\title{
Energy Aspects of Sodium Thiopental Action on Nervous Activity
}

\author{
Yulia S. Mednikova1 ${ }^{*}$ (D), Mikhail K. Kozlov ${ }^{1}$, Alexander N. Makarenko² \\ ${ }^{1}$ Institute of Higher Nervous Activity and Neurophysiology of RAS, Moscow, Russia \\ ${ }^{2}$ Bogomolets National Medical University, Kyiv, Ukraine \\ Email: *mednikova.yu.s@ihna.ru
}

How to cite this paper: Mednikova, Y.S., Kozlov, M.K. and Makarenko, A.N. (2019) Energy Aspects of Sodium Thiopental Action on Nervous Activity. Journal of Behavioral and Brain Science, 9, 33-53. https://doi.org/10.4236/jbbs.2019.92004

Received: January 14, 2019

Accepted: February 17, 2019

Published: February 20, 2019

Copyright $\odot 2019$ by author(s) and Scientific Research Publishing Inc. This work is licensed under the Creative Commons Attribution International License (CC BY 4.0).

http://creativecommons.org/licenses/by/4.0/

\begin{abstract}
Sodium thiopental, used in a narcotic dose, makes it possible to identify the nervous processes that underlie consciousness and establish the causes of its disorder. When studying the cortical EEG activity, the impulses of individual nerve cells and the electromyographic activity of the muscles of the forelimb, it was found that thiopental blocks a number of neuronal reactions requiring energy support: tonic activating reactions to acetylcholine, applied to neurons, cease; the rate of spontaneous neuronal activity drops; the stage of non-specific activation in response to electrocutaneous stimulation disappears. So, thiopental blocks consciousness by significant limitation of the brain energy metabolism. This results in a loss of the adaptive function of the central nervous system. At the same time, glutamatergic excitation, the formation of which does not depend on energy support, is resistant to the action of thiopental. The blocking of the brain's energy supply caused by thiopental, in accordance with its depth, develops in two stages-hypoxic and narcotic. The hypoxic stage is accompanied by hyperactivity in the nervous system, which is manifested by epileptiform discharges on the EEG and powerful unmotivated movement; the narcotic stage is associated with blockade of motor activity and flattening of EEG oscillations. The post-narcotic state associated with the consequence of the hypoxic effect of thiopental leads to the loss of ionic homeostasis and is accompanied by a steady drop in the amplitude of cortical neuron spikes.
\end{abstract}

\section{Keywords}

Sodium Thiopental, Acetylcholine, Hypoxic State, Narcotic Condition, Post-Narcotic Pathology

\section{Introduction}

The study of the narcosis mechanisms on the central nervous system has a long 
history of development. Based on the analysis of various functional changes accompanying the emergence of a narcotic state, the researchers formulated various hypotheses weakly related to each other, in order to substantiate the main effect of the general anesthetics-a cessation of the adaptive function of the brain. The discovery of the central non-specific activating system gave reason to believe that blocking of adaptive behavior, typical for anesthesia, is a consequence of the impairment of this system influence on different regions of the brain, including cortical structures [1]. Later the effect of general anesthetics was linked with their influence on the $\mathrm{GABA}_{\mathrm{A}}$ receptor sites, which prolongs the release of $\mathrm{Cl}^{-}$ions and enhances GABA-ergic inhibition [2]. In addition to ideas about the receptor effect of narcotic drugs, there is also a hypothesis about their hydrophobic effect directly on the bilayer lipid membrane of neurons (specially on zone of ion channel), which leads to the rise in the permeability of neuronal membranes to $\mathrm{K}^{+}$ions [3]. Consequently, the development of the narcotic effect is associated either with the cessation of generalized arousal influence [1], or with an increase in chloride hyperpolarization [2], or with a shunting effect on the exciting currents [3]. But none of these ideas can explain the facts of the activating effect of narcotics of different classes on both activity of individual neurons [4] and behavior [5]. The stimulating effects of anesthesia indicate its indirect effect on the processes that support consciousness, in particular due to the inhibition of energy metabolism. Indeed, it has been found that barbiturates lower glucose energy metabolism by about twofold [6]. Impaired production of macro-energy molecules under the influence of anesthesia can not only affect the functional state of the nervous system, but also cause hypoxic pathology, because the brain is lacking in any reserves of energy resources [7]. The presence of a pathological component in the action of anesthesia suggests that functional changes in the nervous system caused by narcotic drugs may be due to their pathological disorder.

\section{Methods}

\subsection{Experimental Subjects and Operating Procedures}

Animals were treated with observance of recommendation on ethics of work with animals offered by European Communities Council Direction (86/609 EEC) and experimental protocols approved by ethics committees of Institute of Higher Nervous Activity Russian Academy of Sciences. The experiments were carried out on awake rabbits (males aged $1-1.5$ years, weighing $2-2.5 \mathrm{~kg}$ ). Two days before the experiment, under the local anesthesia, an operation was performed to remove the soft tissues of the head in order to expose the bones of the skull. Then, bipolar electrodes were installed over the cortical area of the motor representation of the forelimb (AP0; L2) to record a total electroencephalographic activity, or on the day of the experiment, a hole in the skull was drilled with a $2.5 \mathrm{~mm}$ in diameter according to the same coordinates. After removal of the dura mater the immobile part of the micromanipulator was fixed over the 
aperture for subsequent recording of the spike activity of the neurons. Then the animals were placed on a special platform with a soft fabric cover and gently fixed by the paws. Head movements were limited by specially designed bridle having no traumatic effect on the animal.

In accordance with the recorded parameters, the animals were divided into two groups. In one group (4 rabbits), the total bioelectric activity (EEG) was recorded. In the second group of animals (8 rabbits), impulse activity of nerve cells was investigated.

\subsection{The Introduction of Sodium Thiopental}

Sodium thiopental was administered to animals in the marginal ear vein in a narcotic dose of $30 \mathrm{mg} / \mathrm{kg}$ dissolved in $5 \mathrm{ml}$ of physiological saline. Preliminary experiments have shown that the effects of thiopental last longer than the duration of the narcotic state, and can be recorded several days after the injection. Therefore, the reintroduction of thiopental in experiments with EEG recording was done not earlier than one week after the first injection with the reinstallation of electrodes on the opposite side of the skull. In experiments where the spike activity of neurons was used as a registered parameter, a single neuron was recorded during whole experimental day. Therefore, thiopental was administered two days in a row on the one side and a week later on the other side, in order to be able to register nerve cells twice in the open part of the brain.

\subsection{Recorded Parameters, Equipments, Types of Stimulation}

Total EEG activity, impulse activity of neurons, and electromyogram (EMG) of the extensor muscles of the forelimb were recorded using amplifiers of a universal electrophysiological installation (UEFI-2, Russia). The same device was used for threshold electrocutaneous stimulation of the forelimb (ECS) and for micro-iontophoretic application of mediators to the region of recorded neurons.

The spike activity of neurons was recorded by one of the channels of 3-channel glass microelectrodes (total tip diameter 7.6 - $8 \mu \mathrm{m}$ ) filled with $3 \mathrm{M}$ $\mathrm{NaCl}$ solution (resistance $2-4 \mathrm{M} \Omega$ ). Two other channels were used for micro-iontophoretic injection of the excitatory mediator-glutamate (from $1 \mathrm{M}$ sodium glutamate solution) and the membrane regulator-acetylcholine (from $2 \mathrm{M}$ solution of acetylcholine chloride) to the neurons. The intensity of iontophoretic current for glutamate injection was 25 - $30 \mathrm{nA}$ (negative pole inside the electrode). Acetylcholine was applied with a current of $60-70 \mathrm{nA}$ (positive pole inside the electrode).

A series of 5 rectangular current pulses with a threshold power $(1.5-1.8 \mathrm{~mA})$ and frequency rate of 10/s was used as the ECS. Electrocutaneous stimulation of the forelimb and the registration of EMG activity were carried out by subcutaneous piercing electrodes.

All electrophysiological indicators: EEG, EMG, impulse activity of neurons after amplification were digitized and entered into the Intel (R) Core (TM) 
2DuoCPU computer for further processing using the PowerGraph program (version 3.3; Russia).

\subsection{Protocol of Experiments}

All electrophysiological parameters were studied before, during and after the administration of sodium thiopental. When recording the spike activity of neurons on each experimental day, activity of a single neuron was observed for 5 hours after the introduction of thiopental and its responses to one of the stimulus: electrocutaneous stimulation of the anterior contralateral limb, microiontophoretic administration of acetylcholine or microiontophoretic administration of glutamate were studied. The next day, thiopental was re-injected while recording the activity of another neuron also throughout the whole experimental day. A week later, the same procedure was repeated when registering impulse activity from the other side.

Registration of EEG activity at a constant position of the recording electrodes made it possible to observe the recorded parameter for several days after the 1 st injection of thiopental and the same after the 2nd thiopental, which was administered a week after the first. In narcotic and post-narcotic periods, electrocutaneous stimulation of the forelimb was presented to the animals. A week before the first injection of thiopental, EEG activity was recorded after a control injection of saline for several days in a row.

\subsection{Material Processing and Data Analysis}

EEG was analyzed visually or by calculating Fourier spectra in the form of amplitude spectra on EEG recording segments at different periods before and after injection of sodium thiopental. In the analysis of neuronal spike activity, the frequency of spontaneous spikes, as well as the amount of spike responses caused by exogenously used mediators and sensory stimulation, was determined. At different stages of the thiopental action, the amplitude of the spikes of the recorded neurons was determined. Additional parameter was the spontaneous and induced EMG activity. The reliability of changes in parameters was evaluated by non-parametric statistics [8].

\section{Results}

\subsection{Development of Thiopental Action}

Despite the equal doses of sodium thiopental and the same conditions for administering the drug, electroencephalographic, motor and neuronal parameters differed somewhat in different experiments, apparently due to the different rate of administration of the thiopental solution, different individual sensitivities of animals and due to different functional features of the recorded neurons. In rare cases, for example, a narcotic state did not arise, but changes in the recorded indicators, nevertheless, were observed. The second injection of thiopental (one week after the first) in terms of EEG activity had a more powerful effect. 
At the first stage of the development of anesthesia (10 - 30 s) in half of the experiments, animals displayed the powerful generalized movement, which ceased with the onset of the narcotic state, but had enough time to influence the possibility of further recording the spike activity of neurons. Therefore, long-term registration of spike activity was carried out on a limited number of nerve cells.

The narcotic condition occurred on average within $1-2$ minutes. Figure 1 shows the stages of development of the thiopental effect during the registration of the EEG in the field of motor representation of a forelimb (A) and the concomitant motor response to ECS (B). Starting from the 10th second from the beginning of the introduction of thiopental, changes in the EEG activity are already

\section{Development of narcotic state}

A

1

2

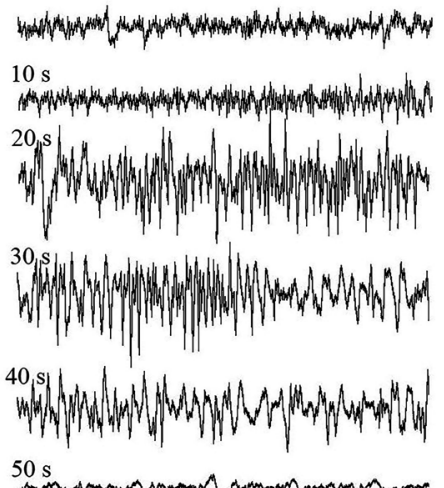

6
B

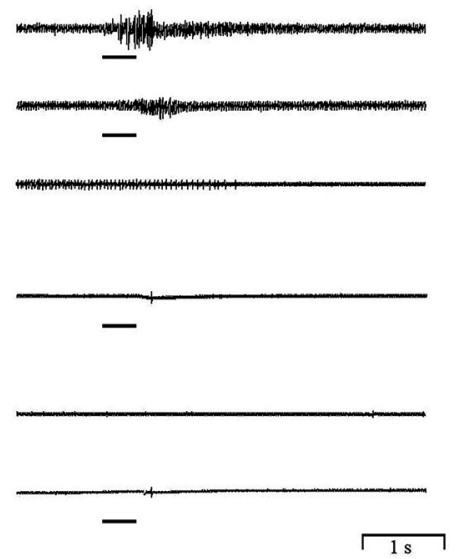

B
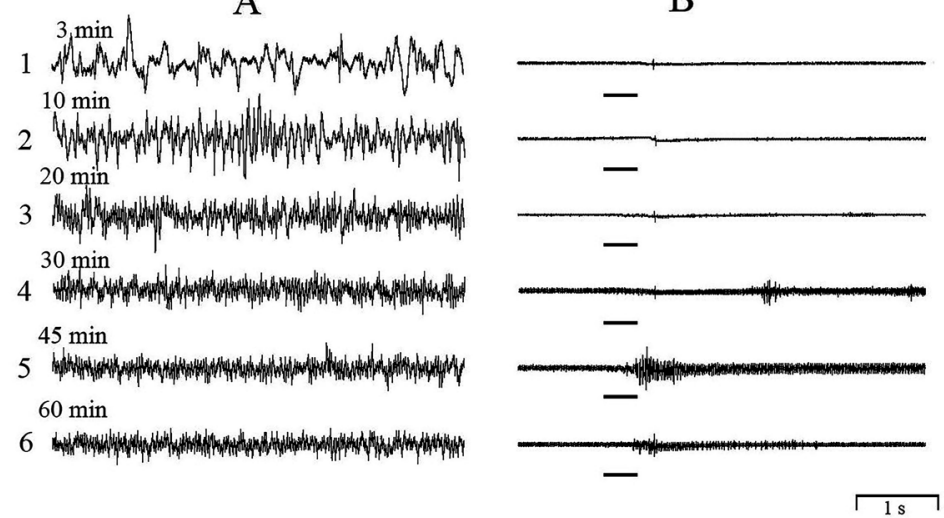

Figure 1. Electroencephalographic indicators of brain activity (EEG) and caused by ECS muscle reactions under the action of sodium thiopental. A-EEG activity in the field of motor representation of the forelimb; B-EMG activity of muscles-extensors of the wrist of the stimulated limb (contralateral to the EEG recording area). I-development of the narcotic state; II-restoration from anesthesia. On I: 1) before the thiopental injection; (2 - 6) at different stages from the beginning of the thiopental injection (in seconds). On II: $(1-6)$ at different stages after stopping the thiopental injection (in minutes). The duration of electrocutaneous stimulation is marked by a line under the records of EMG activity on B. 
occurring (Figure 1, A, 10 s) and in the 20th to 30th seconds are transformed into high-amplitude regular oscillations (Figure 1, A, 20, $30 \mathrm{~s}$ ) and are replaced by a flattening of the EEG activity at the end of the injection of thiopental (Figure 1, A, $50 \mathrm{~s}$ ). The termination of the EMG response to the ECS in a period of more than $30 \mathrm{~s}$ (Figure 1, B, $30 \mathrm{~s}$ ) indicates the onset of the narcotic state. At about the same time the frequency of respiratory movements slows down significantly. Similar changes in EEG and EMG activity were observed in 7 cases out of 8 during the administration of thiopental (four rabbits were used). Only in the absence of narcotic state after thiopental injection the EEG activity did not change the stationary oscillatory form, and evoked EMG reaction did not disappear. In cases where the action of thiopental led to the emergence of early generalized motor activity, simultaneous recording of EEG showed that a powerful EMG activation coincides with the onset of EEG transformation into a high amplitude oscillatory process (Figure 2). Consequently, the development of anesthesia realizes through a stage of hyperactivation in the nervous system, which indicator is a regular epileptiform activity on the EEG, and the result is a powerful motor accompaniment.

The activation stage, characteristic for the first tens of seconds after the start of thiopental injection, can also be detected by short-term increase in the frequency of spike activity of the nerve cells registered in the zone of the motor representation of a forelimb.

In Figure 3, using the example of three neurons, with an individual for each injection of thiopental and recorded on three different experimental days, the stage of a short-term increase in the firing frequency can be seen for $20-30 \mathrm{~s}$ for the N1 neuron, for the 10th s for the N2 neuron and for $10-20 \mathrm{~s}$ for neuron N3. An increase in the spike frequency in all three neurons led to a drop in the spikes amplitude: a very significant for the neurons $\mathrm{N} 1$ and N2 and moderate for the neuron N3. At this stage, the activation period stops and the narcotic effect of thiopental comes. The reason for the substitute in the stages of anesthesia development will be considered further, but in Figure 3, a transition in activity of each neuron is clearly seen: narcosis significantly reduces the frequency of neuronal spike activity and restores (even exceeding) the amplitude of the spikes compared to the original values. For neurons N1 and N3, this happens at the 40th second, and for neuron N2-at the 60th second (Figure 3).

Of the 10 sensorimotor cortex neurons, which activity were registered in the course of the introduction of sodium thiopental to animals, 3 nerve cells were tested by periodic glutamate application, 4-by iontophoretic injection of acetylcholine, spontaneous activity only was recorded in one neuron and the responses to ECS of the contralateral forelimb was recorded in two nerve cells.

Figure 4 demonstrates that when a narcotic condition occurs, simultaneously with the disappearance of the EMG reaction to electrocutaneous stimulation (Figure 4, B, 2 and A, 2), the neuronal activation response to the stimulus disappears, which is accompanied by the already mentioned decrease in the level of spontaneous activity (Figure 4, B, 1 and A, 1). 


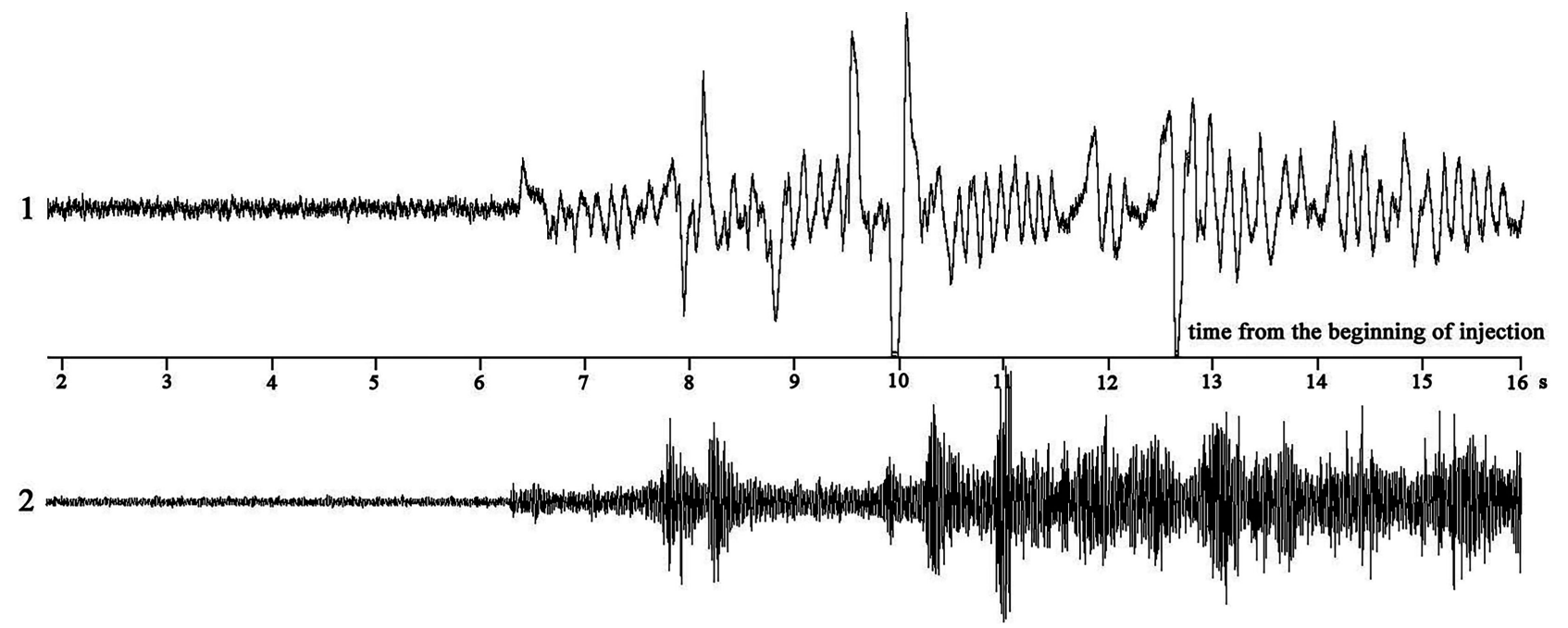

Figure 2. Change in EEG activity (1) and spontaneous electromyographic generalized reaction (2) at the 6th second from the start of thiopental injection.

N1

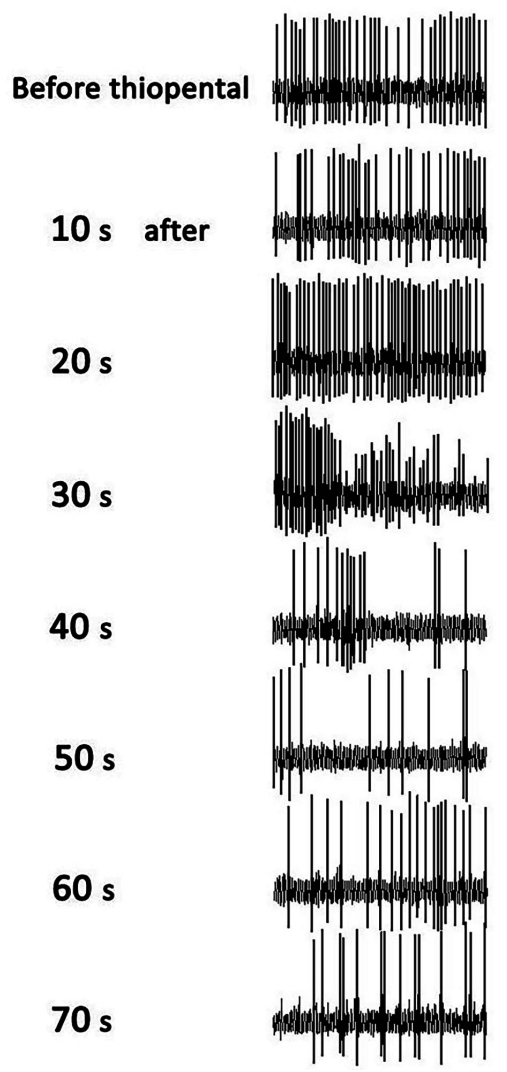

N2

N3
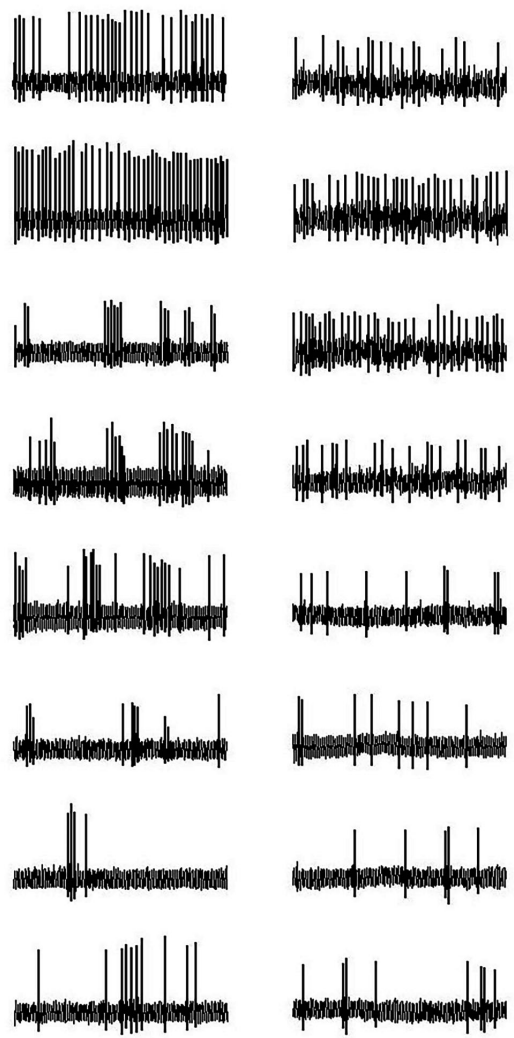

Figure 3. The spike activity of neurons during the introduction of sodium thiopental. N1, N2, N3-the activity of three different neurons registered in the field of motor representation of the forelimb on different experimental days, during the development of the narcotic effect of thiopental, is presented. The initial activity of the neurons is shown on the upper records (before thiopental). In subsequent recordings, the dynamics of spike activity changes in successive periods during the introduction of sodium thiopental (10s - 70s after) is unfolded on 2-second fragments. 
A

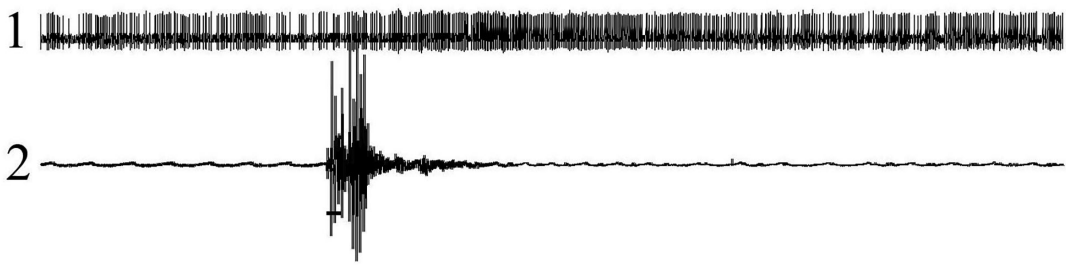

B

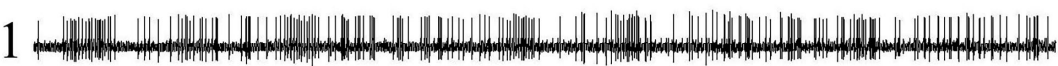

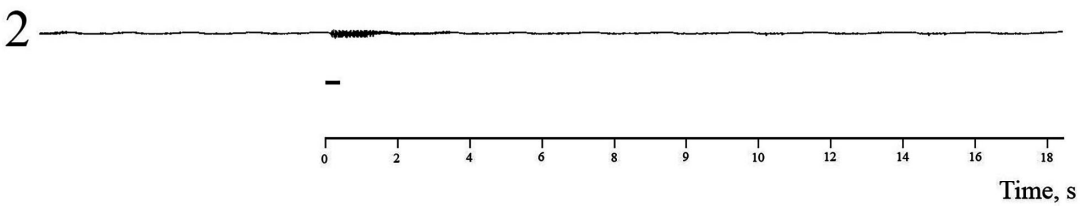

Figure 4. The effect of sodium thiopental on the evoked activity of the neuron of the sensorimotor cortex and on the EMG responses to the electocutaneous stimulation of the forelimb. A-before the introduction of thiopental; B-75 seconds after the start of thiopental injection. 1) neuronal activity-activating tonic spike response on $\mathrm{A}$ and absence of impulse response on B. 2) EMG activity of the forelimb at the same time from the beginning of the of thiopental injection. The duration of the ECS is marked by a rectangle under the records of EMG activity.

In all four neurons tested by iontophoretic application of acetylcholine, the activating reactions to the transmitter completely disappeared with the development of the narcotic effect of thiopental simultaneously with a decrease in the level of spontaneous activity.

Figure 5 presents the records of the activity of two neurons (N1 and N2), that responded with a prolonged activation to the application of acetylcholine in the control (Figure 5, N1, a and N2, a) and of the same neurons at the final stage of thiopental action, when the response to acetylcholine completely disappeared (Figure 5, N1, b and N2, b). It is noteworthy that the long course of response to acetylcholine (Figure 5, N1, a and N2, a) coincides with the dynamics of the tonic neuronal reaction to the ECS (Figure 4, A, 1). Simultaneous their disappearance upon the occurrence of a narcotic state, as well as the drop in the frequency of spontaneous firing, so evidences the identity of mechanisms of origin of these three indicators of neuronal activity.

The activating response of neurons to the iontophoretic injection of glutamate according to the activation excess of spike number above the background level in none of three tested neurons undergo changes upon the onset of a narcotic state, despite the drop in the level of spontaneous activity (Figure 6, N1, a, b and N2, $\mathrm{a}, \mathrm{b})$.

The lack of thiopental blocking of neuronal activating responses to iontophoretic glutamate delivery indicates that thiopental action is not associated with either the receptor or ion mechanisms of the excitatory function of glutamate. 


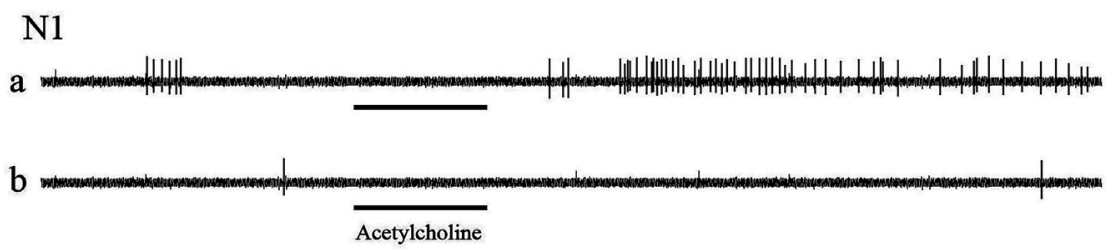

$$
\text { N2 }
$$
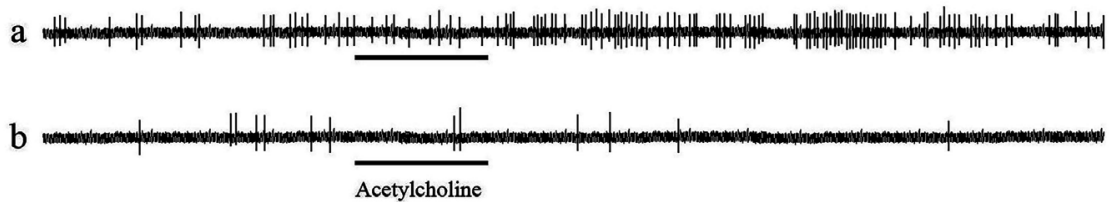

Acetylcholine

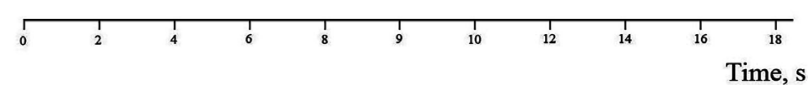

Figure 5. The effect of sodium thiopental on neuronal spike responses to iontophoretic application of acetylcholine. N1 and N2 are two different neurons of the sensorimotor cortex. a-before the introduction of thiopental; b-after $40 \mathrm{~s}(\mathrm{~N} 1)$ and $75 \mathrm{~s}$ (N2) after the start of thiopental injection. The strength of the phoretic current of acetylholine-70 nA. The duration of the phoretic current is marked by a line.

N1

a

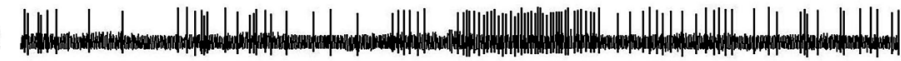

b

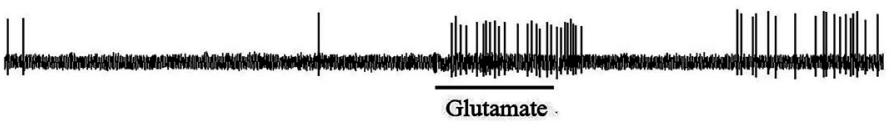

N2

a

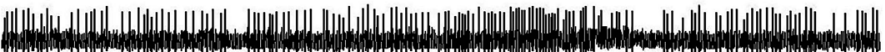

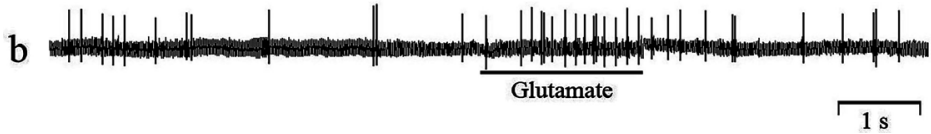

Figure 6. The effect of sodium thiopental on the neuronal spike responses to the iontophoretic application of glutamate. N1 and N2 are two different neurons of the sensorimotor cortex. a-before the introduction of thiopental; b-after $130 \mathrm{~s}$ (N1) and $45 \mathrm{~s}$ (N2) after the start of thiopental injection. The strength of the phoretic current of glutamate- $25 \mathrm{nA}$. The duration of the phoretic current is marked by a line below each recording of neuronal activity.

Judging by the time of onset of changes in different electrographic indicators during a 1 - 2 minute injection of sodium thiopental, the entire period of development of the narcotic state can be divided into two stages (Figure 7).

At the first stage (during the first $30 \mathrm{~s}$ ), there is an increase in spontaneous motor activity, followed by the occurrence of a prolonged high amplitude rhythm 
on the EEG (Figure 7, 1 and 2). This stage is characterized by an increase in spontaneous activity and a decrease in the spikes amplitude in registered neurons, which indicates the development of a pathological process (Table 1). At the second stage of anesthesia (30 - $120 \mathrm{~s}$ ), the adaptive activity of the nervous system is blocked: the neuronal and electromyographic evoked responses disappear (Figure 7, 3), the spontaneous activity of neurons decreases (Figure 7, 4, Table 1), the tonic spike responses of neurons to iontophoretic application of acetylcholine do not reproduce (Figure 7, 5). The onset of these changes under the narcosis action indicates their direct participation in maintaining the function of consciousness.

\subsection{The Post-Narcotic Effect of Thiopental}

15 - 30 minutes after the end of the administration of sodium thiopental, the changes caused by anesthesia in the total cortical EEG, individual neuronal activity and in the motor responses of the animals are restored. This gives reason to believe that the transition to the normalization of the adaptive function of the nervous system is completed. In Figure 1, II, B it can be seen how after a 30 minute recovery period an EMG reaction to electrocutaneous stimulation appears, which was absent in the later stages of thiopental action and immediately after its cancellation (Figure 1, I, B, 4 - 6; II, B, 1 - 3). Powerful changes induced by thiopental in EEG activity (Figure 1, I, A, 3 - 6), are replaced by stationary recordings little different from the reference background activity (Figure 1, II, A, $4-6)$.

Table 1. Changes in the frequency of spontaneous activity and amplitude of the neuronal spikes after injection of thiopental and in the post-narcotic period.

\begin{tabular}{|c|c|c|c|}
\hline \multicolumn{2}{|c|}{ Measured indicator } & Spontaneous activity & Spike amplitude \\
\hline \multicolumn{2}{|c|}{ Before thiopental injection } & $100 \%$ & $100 \%$ \\
\hline \multirow{2}{*}{ Thiopental action } & hypoxic state $10-30 \mathrm{~s}$ & $138 \%^{*}$ & $87.5 \%^{\star}$ \\
\hline & narcotic state $30-120 \mathrm{~s}$ & $29.3 \%{ }^{* *}$ & $106 \%{ }^{*}$ \\
\hline \multirow{9}{*}{ Post-narcotic period } & $3-5 \min$ & $60.9 \%$ & $101.5 \%$ \\
\hline & $10-15 \mathrm{~min}$ & $120 \% *$ & $93.5 \%$ \\
\hline & $30 \mathrm{~min}$ & $110 \% *$ & $75.8 \% \%^{\star *}$ \\
\hline & $1 \mathrm{~h}$ & $141 \%^{*}$ & $58 \% *$ \\
\hline & $1 \mathrm{~h} 30 \mathrm{~min}$ & $128 \%$ & $55.4 \%$ \\
\hline & $2 \mathrm{~h}$ & $150 \%$ & $50.6 \%$ \\
\hline & $3 \mathrm{~h}$ & not established & not established \\
\hline & $4 \mathrm{~h}$ & not established & not established \\
\hline & $5 \mathrm{~h}$ & not established & not established \\
\hline
\end{tabular}

Table 1 shows the average data obtained on ten neurons. The initial (up to thiopental) levels of the frequency of spontaneous activity and amplitude of the spikes are taken as $100 \%$. Indicators of each neuron at different stages after the action of thiopental were compared with their values prior to drug administration. The statistics were relative changes in the values of the indicators (frequency of spontaneous activity $\mathrm{imp} / \mathrm{s}$ or amplitude of spike $-\mu \mathrm{V}$ ) before and at different stages after the action of thiopental. The significance of differences is determined by the Wilcoxon test for pairs of differences $\left({ }^{\star} \alpha \leq 5 \%\right.$; ${ }^{\star *} \alpha \leq 1 \%$ ). 


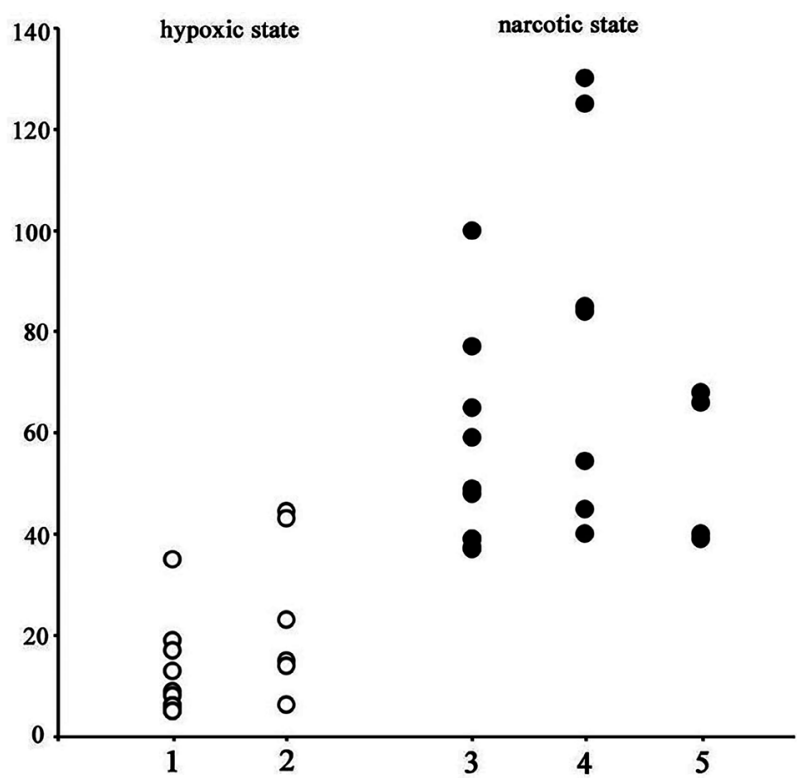

Figure 7. The onset of changes in various electrographic indicators depending on the time after the start of the injection of sodium thiopental. Each circle in the diagrams depicts the time of onset of changes from the beginning of the introduction of thiopental. All cases of occurrence of specific changes are shown. Vertical line-time in seconds. Horizontally: 1) the beginning of a generalized spontaneous movement; 2) the beginning of the emergence of high-amplitude rhythmic activity on the EEG; 3) cessation of adaptive motor responses to electrocutaneous stimulation; 4) achievement of a reliable lowest value of the frequency of spontaneous neuronal activity (Wilcoxon test for pair differences; $\alpha<1 \%)$; 5) termination of neuronal reactions to iontophoretic application of acetylcholine.

But the effect of thiopental does not end with the restoration of consciousness. Thus, Table 1 summarizes the data for all registered neurons, from which it follows that a sharp drop in spike frequency during the narcotic period quickly develops in the opposite direction, and by the 30-minute post-narcotic stage the firing rate significantly exceeds the initial level, and the spike amplitude decreases significantly.

Figure 8 shows the post-narcotic growth of background neuronal spike frequency (Figure 8, B, 2, 3) and a gradual drop in the amplitude of the spike in recovery period, when the activation reaction to acetylcholine already reproduced stable (Figure 8, B, 1 - 3) while its absence during thiopental action (Figure 8, A, 2, 3). Consequently, after the restoration of the adaptive function of the brain, the effect of thiopental does not completely disappear. Residual effects can be registered by the continued decrease in the spike amplitude of the neuron presented in Figure 8 (B, 4 and C, 1, 2) and in all other neurons (see Table 1) registered in the post-narcotic period. As a result of falling in spikes amplitude, the spikes ceased to be distinguished from the background noise and did not appear up to the end of the experimental day. Only in rare cases the amplitude was partially restored (Figure 8, C, 3), but did not persist for a long time and disappeared again after half an hour of registration. 

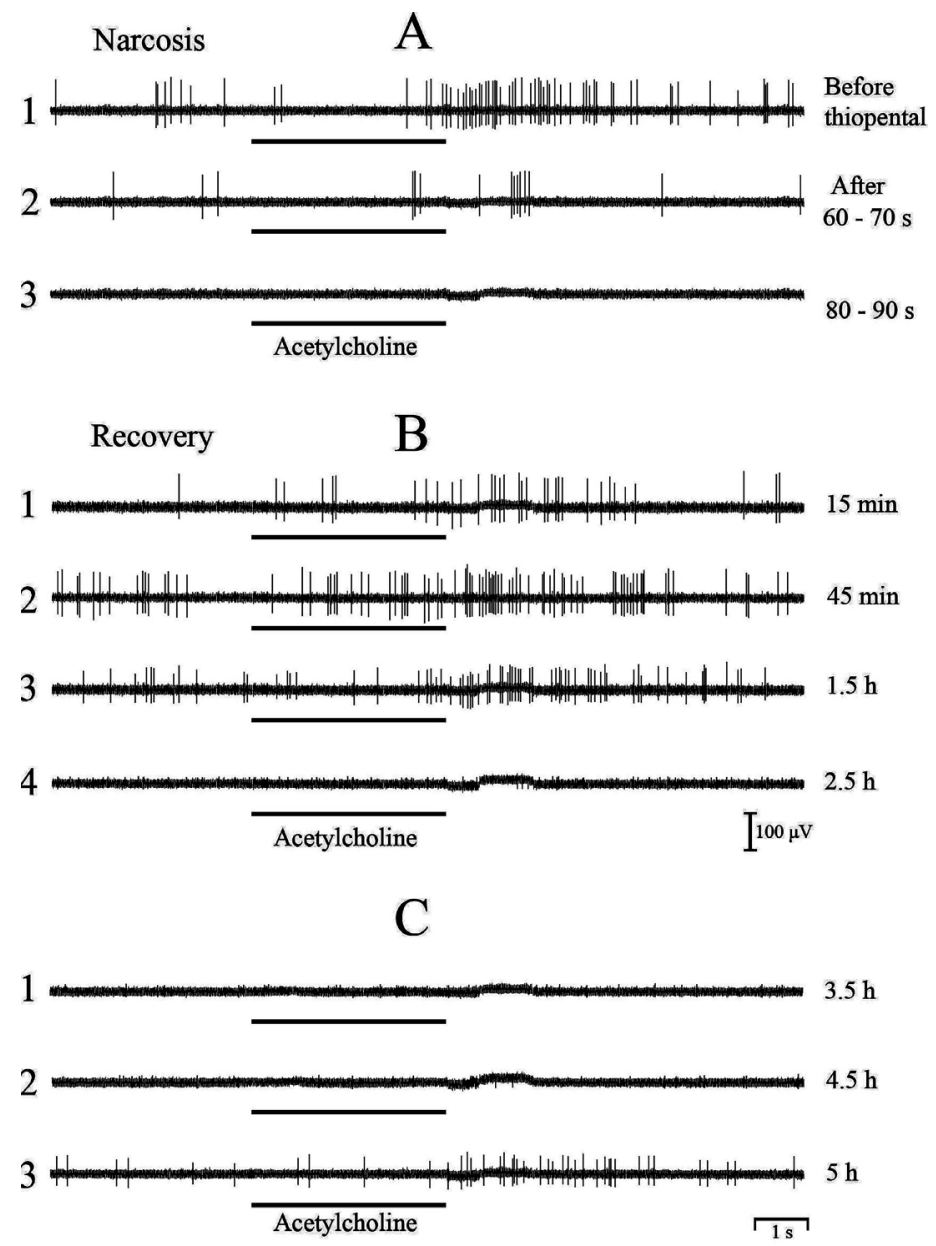

Figure 8. Background activity of the sensorimotor cortex neuron and spike response to the iontophoretic application of acetylcholine during narcotic and post-narcotic periods. A-in the period of development of the narcotic state: 1 -before the thiopental injection; 2, 3-60 - $70 \mathrm{~s}$ and $80-90 \mathrm{~s}$ after the start of the thiopental injection. B-the first stage of the recovery period: $(1-4)$ up to 2.5 hours after the end of the thiopental injection; C-the second stage of the recovery period: $(1-3)$ after 3.5 - 5 hours after the end of the thiopental injection. The current strength of phoresis of acetylcholine is $70 \mathrm{nA}$. The time of phoretic current of acetylcholine is indicated by a line under each records of neuron activity.

The second evidence of the altered state of the nervous system in the post-narcotic period is the shift of amplitude spectra on the EEG records to the higher frequency region both immediately after the termination of the thiopental (Figure 9, 2), and after 1 h. 45 minutes (Figure 9, 3). It also should be pay attention to the considerable inconstancy of the structure of the spectrum of EEG amplitudes, built on different fragments during registration in the same period after the action of anesthesia (Figure 9, 2 and 3) in contrast to the consistently reproducible form before thiopental action (Figure 9, 1). Full recovery of EEG frequency content did not occur just within a week after application of thiopental (Figure 9, 4 and 5). Injection of saline did not lead to a change in the EEG spectra either immediately after exposure or after a few days. 

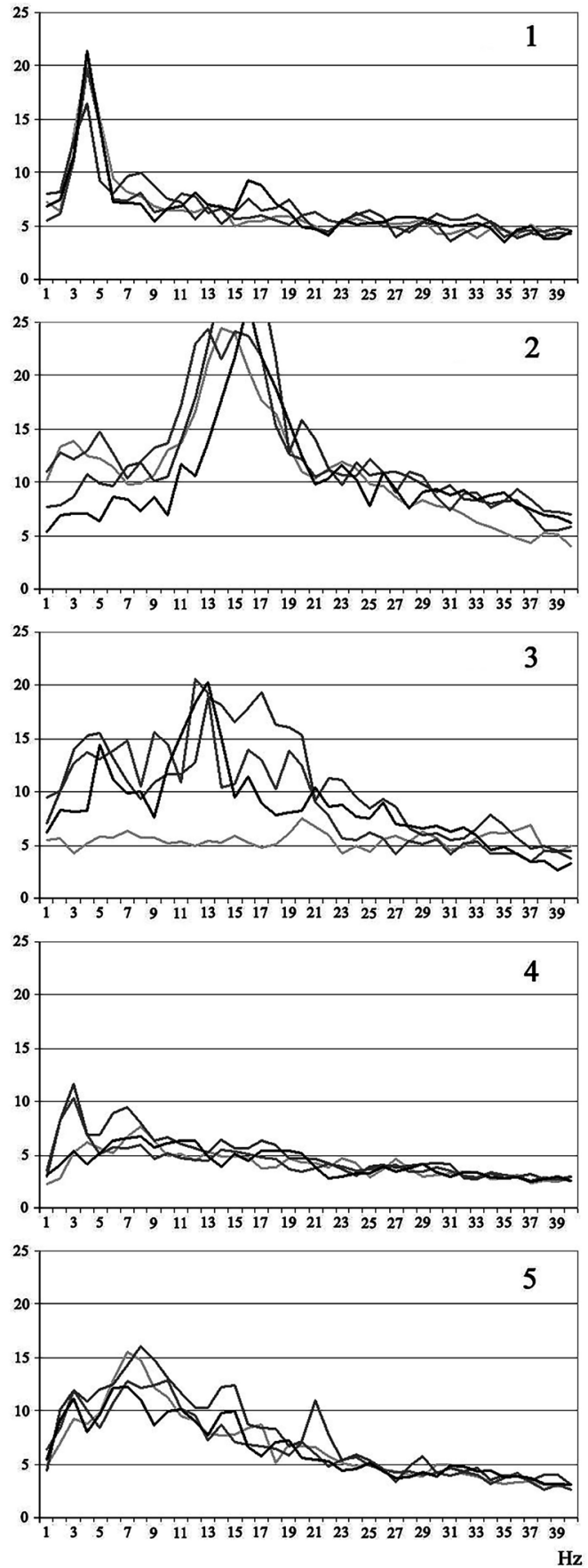

Figure 9. Fourier spectra (presented in the amplitude scale) for EEG segments before the action of thiopental and in different periods of the post-narcotic state. 1) before the introduction of thiopental; 2) 5 minutes after the end of the thiopental injection; 3) 1 hour $45 \mathrm{~m}$ after the end of the thiopental injection; 4) 3 days after administration of thiopental; 5) 6 days after the administration of thiopental. On EEG records corresponding to different states, homogeneous fragments were selected, with a duration of 5 - 15 s. The Fourier transform counting epoch was chosen with a duration of $1 \mathrm{~s}$ with averaging of the result over a sliding window with the corresponding shift. In each graphic window (1 - 5), a superposition of the obtained spectra is displayed for all fragments selected on the EEG record corresponding to a particular state. The abscissa is frequency, $\mathrm{Hz}$, and the ordinate is amplitude, conventional units. 
Thus, by analyzing the spike activity of neurons and EEG spectra it should be concluded that the post-narcotic state for a long time changes the initial parameters of the activity of the nervous system. We believe that the reason for these changes is related to the period of the first 30 seconds of the thiopental action. The high-amplitude activity on the EEG arising in this period is associated with a decrease in the amplitude of the spikes of nerve cells (Figure 3), which is a sign of the development of pathology. In the post-narcotic period, $30-60$ minutes after the end of the thiopental injection, it can be also observed a massive appearance of bursts of high-amplitude rhythmic oscillations on the EEG (Figure $10, B, 5-8)$, and in the activity of neurons-a decrease in the spikes amplitude with increasing their frequency (Figure 8, B; Table 1).

Figure 10, A presents data on the four presentation of the first thiopental and the four presentation of the second thiopental in comparison with four injections of saline.

Figure 10, A demonstrates the averaged time duration of fragments of high-amplitude EEG activity at different stages of the post-narcotic period. Despite the fact that flashes of high-amplitude activity can be observed in the control, the effect of thiopental significantly increases their appearance, especially after its re-introduction a week after the first injection (Figure 10, A, Thiopental 2). Increased generation of high-amplitude flashes can be registered with gradual attenuation on the second and third day. Recovery to the control level occurs on the 4th - 6th day. After the first thiopental recovery of EEG activity occurs earlier (on the 2nd - 3rd day).

Experiments have shown that the post-narcotic state and the first few tens of seconds from the beginning of the introduction of thiopental are characterized by similar changes in electrographic indices. They consist in the appearance of high-amplitude rhythmic activity on the EEG, an increase in the spontaneous activity of neurons, and a decrease in the amplitude of their spikes. At the same time, the length of the post-narcotic state stretches for several hours and even days, whereas the initial period of the action of thiopental is quickly interrupted by the development of a narcotic state.

\section{Discussion}

\subsection{Energy Aspect of Consciousness}

For many decades, the mechanism of action of central anesthetics is interpreted both from the standpoint of non-specific (non-receptor) [1] [3] and specific (receptor) [2] [9] concepts. At the same time, the blocking of consciousness, the main effect of narcotic drugs, requires consideration of once more aspect, the energetic aspect, since maintaining consciousness is an active process. Performing any adaptive function is accompanied by energy consumption, increased energy metabolism and local cerebral blood flow [10] [11]. Despite the stable opinion that the basic energy needs of the brain are associated with the restoration of ionic imbalance disturbed as a result of an increase in spike activity during 
A
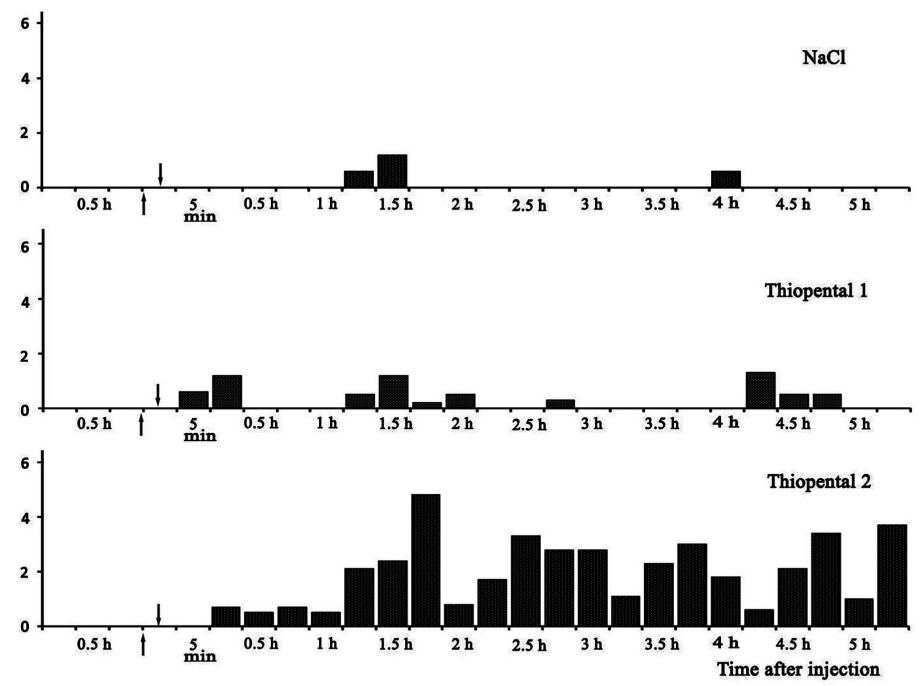

B

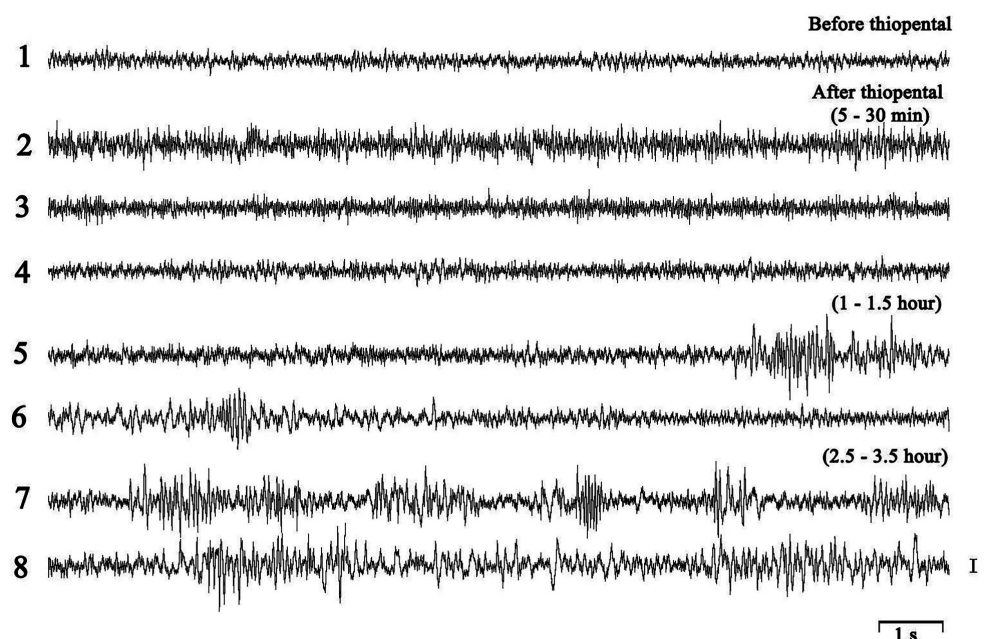

Figure 10. Post-narcotic development of high-amplitude rhythmic oscillations in the EEG. A-the total duration of periods of high-amplitude rhythmic activity, determined on a 40-second interval every 15 minutes of EEG recording and averaged over all four animals: after intravenous saline, the 1st thiopental injection, and the 2 nd thiopental injection (a week after the first). The abscissa is the time after the injection of thiopental (or $\mathrm{NaCl}$ solution); the ordinate is the average duration of periods of high-amplitude activity (in the 40-second interval). The arrows indicate the duration of each injection. B-examples of recording EEG activity at different stages after the 2nd injection of sodium thiopental: 1) before the action of thiopental; 2, 3, 4) respectively, 5, 15, 30 minutes after the end of the thiopental injection: stationary EEG activity; 5, 6) 1 and 1.5 hours after the end of the thiopental, respectively: the beginning of the formation of high-amplitude flashes of activity on the EEG; 7, 8) 2.5 and 3.5 hours after the end of the action of thiopental, respectively: areas of constant repetition of high-amplitude rhythmic potentials on the EEG.

excitation [12] [13], there are absolutely no ideas about the energetics of the own adaptive increasing impulsation. In other words, the high adaptive abilities of warm-blooded animals may be related primarily to the ability of their neurons to 
form very high-frequency spike sequences per stimulus, which cannot be generated by cold-blooded neurons. These evolutionary advantages, apparently, require significant energy costs, five times exceeding the energy budget of a cold-blooded brain [7]. But at the same time with the opening advantages, we inevitably acquire serious problems-a high dependence of the brain on energy supply and sensitivity to anesthesia.

Adaptive growth of spike activity is not related to enhance of the specific neuronal response to the stimulus, which is a signal-determined, energy-independent process, but it related to increase the frequency of spontaneous activity associated with the action of the stimulus. The growth of spontaneous activity is regulated by the release of acetylcholine from the cholinergic nuclei of the brain [14]. This reaction forms the non-specific component of the response to the stimulus, and it is just the component that determines whether the behavior reaction to the stimulus will occur [14]. Therefore, the role of acetylcholine in the formation of the adaptive function of the brain is so great-from maintaining the level of consciousness to memory formation [15] [16]. The ionic mechanism of acetylcholine action, realized through the $\mathrm{M}$-cholinergic receptors consists in a global blockade of the $\mathrm{K}^{+}$channels of the neuronal membrane [17] [18]. This effect leads to an increase in membrane resistance by no more than $10 \%$ [19], but this is sufficient for a multiple increase in the efficiency of the conductive function of dendrites [20] - a high-frequency growth of spontaneous neuronal activity occurs, an adaptive response to the stimulus is formed, and after it-comes the consciousness.

The M-cholinergic response of the brain, responsible for the global change in the membrane properties of neurons, is a very energy-dependent process [21]. Therefore, when considering the mechanism of action of central anesthetics, their influence on the energy metabolism of the brain comes to the fore.

\subsection{Hypoxic and Harcotic Effects of Thiopental}

According to positron emission tomography (PET), barbiturates reduce cerebral glucose metabolism by about 2 times [6]. Similar data on the fall in energy metabolism of the brain were obtained when assessing oxygen consumption under the influence of thiopental anesthesia: thiopental at a narcotic dose reduced brain consumption of oxygen by $1 / 3$ compared with the control level [22]. Based on the data obtained, the researchers concluded that the decrease in energy metabolism during anesthesia is due to inhibition of the mitochondrial respiratory chain, which was confirmed at the biochemical level [23] [24].

For comparison, it can be pointed out that in the nuclear zone of a stroke the speed of cerebral blood flow decreases by 5 times, and in the zone of ischemic penumbra, by about 2 times [25]. Therefore, the effect of anesthesia should have much in common with the hypoxic state of the brain.

When analyzing the energy needs of the nervous system, it should be kept in mind that different metabolic reactions due to their different rate will depend 
differently on the energy supply. In the case of partial blocking of energy metabolism (for whatever reason it may occur), the highest-speed reaction will have an advantage for access to macro-energy adenosine triphosphate (ATP) compounds. In thermobiological experiments conducted on the slices of the sensorimotor cortex of guinea pigs, it was found that at temperatures above $36^{\circ} \mathrm{C}$ there is sharply increase of the rate of cholinergic reaction [26], that is why conditions arise for the competitive interaction between energy-dependent brain processes [26]. The reason for the development of these competition relations is the inadequate supply of brain tissue with energy substrate under conditions of artificial incubation. There is a hypoxic state in which the M-cholinergic reaction will have an advantage due to its high speed at $36^{\circ} \mathrm{C}$, and other metabolic reactions, including $\mathrm{Na}^{+}, \mathrm{K}^{+}$-ATPase, will suffer from energy hunger, which will immediately affect the amplitude of the spikes of nerve cells-it will begin to decline progressively [27].

Something like this should happen under the action of sodium thiopental, which blocks the energy metabolism of neurons, at a temperature of $39^{\circ} \mathrm{C}$, which is typical for the brain of alive rabbits. At the first seconds of thiopental injection, a hypoxic state occurs in the nervous tissue. The M-cholinergic reaction of the brain, due to its speed advantage, satisfies its energy needs by inhibiting the activity of transmembrane ion transporters. This leads to a rapid drop in the spikes amplitude of nerve cells (Figure 3;20 - 30 s) and a massive leakage of $\mathrm{K}^{+}$ ions from neurons [3]. An increase in the extracellular content of $\mathrm{K}^{+}$ions slightly shifts the membrane potential (MP) towards depolarization, which can be seen from the flattening of the EEG oscillations in the late stages of the thiopental (Figure 1, A, 6). The gradual flattening of EEG activity is also observed when registering from the zone of ischemic penumbra during the period of cerebral stroke formation [25] [28]. This analogy demonstrates the pathological basis of the anesthesia action.

A respite occurs when, under the influence of thiopental, energy metabolism decreases so much that the M-cholinergic process becomes impossible (Figure 5). Huge energy resources serving this reaction can now be used to maintain the viability of the nervous tissue. Even in conditions of a lack of energy, the amplitude of neuronal spikes is restored (Figure 3,60 - 70 s), but at the same time, nerve cells lose ability to perform their specific function-to provide the adaptive activity of the organism. A narcotic condition occurs, in which, simultaneously with the blockade of the activation reaction to acetylcholine, a non-specific neuronal reaction to the ECS disappears (Figure 4) and electromyographic responses abolish; the level of spontaneous neuronal activity falls (Figure 3 and Figure 4, Figure 5, Figure 7). These data confirm that the adaptive function of the brain is an active process and requires for its implementation in warm-blooded large energy support, far exceeding the energy needs of ion carriers.

At the same time, the responses of neurons to micro-iontophoretic glutamate delivery did not change under the influence of thiopental (Figure 6), which in- 
dicates the energy independence of glutamatergic depolarization.

\subsection{Post-Narcotic Pathology}

Vigorous events occurring in the nervous system with the introduction of thiopental end rather quickly after its cancellation: within 30 minutes, as a rule, the responses to acetylcholine (Figure 8, B) and motor reactions to electrocutaneous stimulation (Figure 1, II, B, 4) recover; EEG acquires a characteristic form of a stationary oscillatory process (Figure 1, II, A, $4-6$ ). At the same time, 30 - 60 minutes after the cessation of the thiopental injection, the amplitude of the spikes of nerve cells begins to fall again, as happened at the first stage of thiopental introduction, but in the absence of a hypoxic state. This is due, apparently, to the fact that $\mathrm{K}^{+}$ions, in large numbers leaving nerve cells due to the restriction of $\mathrm{Na}^{+}, \mathrm{K}^{+}$-ATPase activity at the initial stage of thiopental action, decrease the ratio of ion concentrations on both sides of the membrane, which leads to a small shift of the MP towards the depolarization. And since $\mathrm{K}^{+}$channels are potential-dependent, the constant depolarization, little affected by rapid elimination, leads to an additional opening of $\mathrm{K}^{+}$channels, an even greater yield of $\mathrm{K}^{+}$from the cells and an even greater shift of the MP towards the depolarization. So, a self-sustaining process of accumulation of the external concentration of $\mathrm{K}^{+}$ions occurs with a gradual decrease in the spikes amplitude-the greater the deeper was the initial ionic imbalance and the longer was the period for the unfolding of the pathological state (Figure 8, B, C).

There are two ways out of this situation: either the restored $\mathrm{Na}^{+}, \mathrm{K}^{+}$-ATPase activity (and even enhanced due to an excess of substrate) and the glial elements of the brain eventually normalize the ionic homeostasis, or the neurons will die one after the other, which can lead to serious post-narcotic disorders until death of the organism. The same effect of persistent post-ischemic accumulation of extracellular $\mathrm{K}^{+}$can explain the massive neuronal death that was delayed (2 days) after 2-hour focal ischemia [28]. Thus, anesthesia can be considered as a particular case of short-term hypoxic exposure.

Based on this conclusion, it is possible to imagine the cause of all the narcotic and post-narcotic phenomena observed in electrophysiological indicators under the action of sodium thiopental:

1) The impairment of consciousness is associated with a deep suppression of the energy metabolism of neurons by thiopental, as a result of which the M-cholinergic energy-dependent reaction is blocked (Figure 5 and Figure 7, Figure 8).

2) Since acetylcholine regulates the level of spontaneous activity and the nonspecific component of responses to sensory stimulation, the narcotic effect is associated with a drop in the frequency of neuronal spike activity (Figure 3 and Figure 7), with the termination of the neuronal activating response to electrocuteneous stimulation (Figure 4) and an electromyographic response (Figure 1 and Figure 4, Figure 7).

3) The initial (hypoxic) effect of thiopental is associated with the restriction of $\mathrm{Na}^{+}, \mathrm{K}^{+}$-ATPase activity and the rapid accumulation of $\mathrm{K}^{+}$ions in the extracellu- 
lar space. As a result, the depth of post-activation hyperpolarization decreases and epileptiform activity appears on the EEG (Figure 1 and Figure 2) because of prolongation of any activating phase. At the same time, due to the concentration limitation of $\mathrm{K}^{+}$permeability and growth of membrane resistance, the spontaneous activity of neurons increases (Figure 3), resulting from more efficient conduction of excitation from dendrites.

4) The result is the development of hyperactivity in the nervous system and the emergence of powerful generalized spontaneous movement (Figure 2). This unmotivated hyperactivity has a passive origin-it is not associated with the work of the adaptive mechanism of the brain, but only with an increase in the external concentration of $\mathrm{K}^{+}$ions, which appears in accordance with the concentration gradient.

5) A further increase in the extracellular content of $\mathrm{K}^{+}$ions occurs already with the normalization of the activity of $\mathrm{Na}^{+}, \mathrm{K}^{+}$-ATPase in the postnarcotic period and develops due to the potential-dependent nature of the $\mathrm{K}^{+}$channels and small depolarization, which originated during the hypoxic period of thiopental action. The spontaneous impulses of neurons increase again, followed by a decrease in the amplitude of the spikes (Figure 8, Table 1), and a large number of episodes of epileptiform activity appear an hour after the termination of the thiopental (Figure 10).

6) The post-hypoxic period associated with impaired ionic homeostasis of neurons can last for a long time (hours and even days or more), as evidenced by the persistent shift of the EEG spectra to the high-frequency region (Figure 9).

\section{Conclusion}

Our study has shown that the action of sodium thiopental is associated with the impairment of the energy metabolism of neurons; therefore it causes a pathological effect on the nervous system. During the development of thiopental action the hypoxic state of the brain tissue is followed by the narcotic one. Such a transition is related to a high rate of M-cholinergic reaction in warm-blooded animals and high dependence of this reaction on the energy supply. The consequence of the pathological effect of thiopental is a development of a post-narcotic condition-a long-lasting loss of the ion homeostasis of nerve cells.

\section{Acknowledgements}

This research was supported by the Russian Academy of Sciences.

\section{Conflicts of Interest}

The authors declare no conflicts of interest regarding the publication of this paper.

\section{References}

[1] Magoun, H.W. (1958) The Waking Brain. Charles Thomas Publisher, Springfield. 
https://doi.org/10.1037/11149-000

[2] Brickley, S.G., Franks N.P. and Wisden, W. (2018) Modulation of GABA Receptor $^{2}$ Function and Sleep. Current Opinion in Physiology, 2, 51-57.

https://doi.org/10.1016/j.cophys.2017.12.011

[3] Ries, C.R. and Puil, E. (1999) Ionic Mechanism of Isoflurane's Action on Thalamocortical Neurons. Journal of Neurophysiology, 81, 1802-1809. https://doi.org/10.1152/jn.1999.81.4.1802

[4] Wan, X. and Puil, E. (2002) Pentobarbital Depressant Effects Are Independent of GABA Receptors in Auditory Thalamic Neurons. Journal of Neurophysiology, 88, 3067-3077. https://doi.org/10.1152/jn.00365.2002

[5] Shvets-Ténéta-Guriǔ, T.B. and Ivanova-Anninskaia, E.L. (1997) Changes in the Oxidation-Reduction Potential of the Rat Brain during Nembutal Anesthesia. I.P. Pavlov Journal of Higher Nervous Activity, 47, 523-531. (In Russian)

[6] Blacklock, J.B., Oldfield, E.H., Di Chiro, G., Tran, D., Theodore, W., Wright, D.C. and Larson, S.M. (1987) Effect of Barbiturate Coma on Glucose Utilization in Normal Brain versus Gliomas. Positron Emission Tomography Studies. Journal of Neurosurgery, 67, 71-75. https://doi.org/10.3171/jns.1987.67.1.0071

[7] Ivanov, K.P. (2004) Principles of Energetics in an Organism: Theoretical and Applied Aspects. Energy Resources of Organism and Physiology of Survival. Vol. 4, Nauka, St. Petersburg. (In Russian)

[8] Sachs, L. (1972) Statistische Auswertungsmethoden. Springer Verlag, Berlin, Heidelberg, New York. https://doi.org/10.1007/978-3-662-10037-0

[9] Mihic, S.J., Ye, Q., Wick, M.J., Koltchine, V.V., Krasowski, M.D., Finn, S.E., Mascia, M.P., Valenzuela, C.F., Hanson, K.K., Greenblatt, E.P., Harris, R.A. and Harrison, N.L. (1997) Sites of Alcohol and Volatile Anaesthetic Action on GABA(A) and Glycine Receptors. Nature, 389, 385-389. https://doi.org/10.1038/38738

[10] Conejo, N.M., González-Pardo, H., Vallejo, G. and Arias, J.L. (2007) Changes in Brain Oxidative Metabolism Induced by Water Maze Training. Neuroscience, 145, 403-412. https://doi.org/10.1016/j.neuroscience.2006.11.057

[11] Stender, J., Kupers, R., Rodell, A., Thibaut, A., Chatelle, C., Bruno, M.-A., Gejl, M., Bernard, C., Hustinx, R., Laureys, S. and Gjedde, A. (2015) Quantitative Rates of Brain Glucose Metabolism Distinguish Minimally Conscious from Vegetative State Patients. Journal of Cerebral Blood Flow \& Metabolism, 35, 58-65.

https://doi.org/10.1038/jcbfm.2014.169

[12] Laughlin, S.B. and Attwell, D. (2004) Neural Energy Consumption and the Representation of Mental Events. In: Shulman, R.G. and Rothman, D.L., Eds., Brain Energetics and Neuronal Activity. Applications to fMRI and Medicine, John Wiley \& Sons, Ltd., Chichester, 111-124.

[13] Wong-Riley, M.T.T. (1989) Cytochrome Oxidase: An Endogeneous Metabolic Marker for Neuronal Activity. Trends in Neurosciences, 12, 94-101. https://doi.org/10.1016/0166-2236(89)90165-3

[14] Acquas, E., Wilson, C. and Fibiger, H.C. (1996) Conditioned and Unconditioned Stimuli Increase Frontal Cortical and Hippocampal Acetylcholine Release: Effects of Novelty, Habituation, and Fear. Journal of Neuroscience, 16, 3089-3096. https://doi.org/10.1523/JNEUROSCI.16-09-03089.1996

[15] Bentley, P., Driver, J. and Dolan, R.J. (2011) Cholinergic Modulation of Cognition: Insights from Human Pharmacological Functional Neuroimaging. Progress in Neurobiology, 94, 360-388. https://doi.org/10.1016/j.pneurobio.2011.06.002

[16] Sarter, M. and Bruno, J.P. (2000) Cortical Cholinergic Inputs Mediating Arousal, 
Attentional Processing and Dreaming: Differential Afferent Regulation of the Basal Forebrain by Telencephalic and Brainstem Afferents. Neuroscience, 95, 933-952. https://doi.org/10.1016/S0306-4522(99)00487-X

[17] Krnjević, K., Pumain, R. and Renaud, L. (1971) The Mechanism of Excitation by Acetylcholine in the Cerebral Cortex. Journal of Physiology (L), 215, 247-268. https://doi.org/10.1113/jphysiol.1971.sp009467

[18] McCormick, D.A. and Prince, D.A. (1986) Mechanisms of Action of Acetylcholine in the Guinea-Pig Cerebral Cortex in Vitro. Journal of Physiology $(L), 375,169-194$. https://doi.org/10.1113/jphysiol.1986.sp016112

[19] ffrench-Mullen, J.M.H., Hori, N., Nakanishi, H., Slater, N.T. and Carpenter, D.O. (1983) Asymmetric Distribution of Acetylcholine Receptors and M Channels on Prepyriform Neurons. Cellular and Molecular Neurobiology, 3, 163-181. https://doi.org/10.1007/BF00735280

[20] Mednikova, Yu.S., Kopytova, F.V. and Zhadin, M.N. (2010) Spontaneous Activity of Cortical Neurons in Vitro and Its Regulation by Acetylcholine. Neuroscience and Behavioral Physiology, 40, 986-992. https://doi.org/10.1007/s11055-010-9357-6

[21] Godfraind, J.M., Kawamura, H., Krnjević, K. and Pumain, R. (1971) Actions of Dinitrophenol and Some Other Metabolic Inhibitors on Cortical Neurons. Journal of Physiology (L), 215, 199-222. https://doi.org/10.1113/jphysiol.1971.sp009465

[22] Mcllwain, H. (1959) Biochemistry and the Central Nervous System. London: A. and J. Churchill.

[23] Luk'yanova, L.D. (1997) Bioenergetic Hypoxia: Definition, Mechanisms, and Methods of Correction. Bulletin of Experimental Biology and Medicine, 124, 835-843. https://doi.org/10.1007/BF02446979

[24] Makarenko, A.N. and Savosko, S.I. (2016) Influence of Thiopental Sodium on Energy Metabolism in the Brain. Journal of Medical and Biological Problems, 1, 56-59. (In Russian)

[25] Ismagilov, M.F. (2005) Ischemic Cerebral Stroke: Terminology, Epidemiology, Principles of Diagnosis, Pathogenetic Subtypes, Treatment of the Acute Period of the Disease. V.M. Bekhterev Neurological Bulletin, 37, 67-76. (In Russian)

[26] Mednikova, Yu.S. and Pasikova, N.V. (2005) The Temperature Sensitivity of the Cholinergic Responses of Cortical Neurons in the Guinea Pig Brain. Neuroscience and Behavioral Physiology, 35, 615-621. https://doi.org/10.1007/s11055-005-0101-6

[27] Pasikova, N.V., Mednikova, Yu.S. and Averina, I.V. (2011) Thermal Regulation of Spontaneous Spike Frequency and Concomitant Changes in the Amplitude of Spikes from Cortical Neurons. Neuroscience and Behavioral Physiology, 41, 696-702. https://doi.org/10.1007/s11055-011-9474-x

[28] Lipton, P. (1999) Ischemic Cell Death in Brain Neurons. Physiological Reviews, 79, 1431-1568. https://doi.org/10.1152/physrev.1999.79.4.1431 